\title{
Genetic ablation of FLRT3 reveals a novel morphogenetic function for the anterior visceral endoderm in suppressing mesoderm differentiation
}

\author{
Joaquim Egea, ${ }^{1,5,8}$ Christian Erlacher, ${ }^{1,5}$ Eloi Montanez, ${ }^{2}$ Ingo Burtscher, ${ }^{3}$ Satoru Yamagishi, ${ }^{1}$ \\ Martin HeB, ${ }^{4}$ Falko Hampel, ${ }^{1}$ Rodrigo Sanchez, ${ }^{1,6}$ Maria Teresa Rodriguez-Manzaneque, ${ }^{2}$ \\ Michael R. Bösl, ${ }^{1}$ Reinhard Fässler, ${ }^{2}$ Heiko Lickert, ${ }^{3}$ and Rüdiger Klein ${ }^{1,7}$ \\ ${ }^{1}$ Department of Molecular Neurobiology, Max-Planck Institute of Neurobiology, 82152 Martinsried, Germany; ${ }^{2}$ Department \\ of Molecular Medicine, Max-Planck Institute for Biochemistry, 82152 Martinsried, Germany; ${ }^{3}$ Institute of Stem Cell \\ Research, Helmholtz Zentrum München, 85764 Neuherberg, Germany; ${ }^{4}$ Biozentrum der LMU Biology, \\ 82152 Martinsried, Germany
}

\begin{abstract}
During early mouse development, the anterior visceral endoderm (AVE) secretes inhibitor and activator signals that are essential for establishing the anterior-posterior (AP) axis of the embryo and for restricting mesoderm formation to the posterior epiblast in the primitive streak (PS) region. Here we show that AVE cells have an additional morphogenetic function. These cells express the transmembrane protein FLRT3. Genetic ablation of FLRT3 did not affect the signaling functions of the AVE according to the normal expression pattern of Nodal and Wnt and the establishment of a proper AP patterning in the epiblast. However, FLRT3 ${ }^{-/-}$embryos showed a highly disorganized basement membrane (BM) in the AVE region. Subsequently, adjacent anterior epiblast cells displayed an epithelial-to-mesenchymal transition (EMT)-like process characterized by the loss of cell polarity, cell ingression, and the up-regulation of the EMT and the mesodermal marker genes Eomes, Brachyury/T, and FGF8. These results suggest that the AVE acts as a morphogenetic boundary to prevent EMT and mesoderm induction in the anterior epiblast by maintaining the integrity of the BM. We propose that this novel function cooperates with the signaling activities of the AVE to restrict EMT and mesoderm induction to the posterior epiblast.
\end{abstract}

[Keywords: EMT; FLRT3; anterior visceral endoderm; basement membrane; epithelial-to-mesenchymal transition; morphogenesis]

Supplemental material is available at http://www.genesdev.org.

Received May 9, 2008; revised version accepted September 29, 2008.

Gastrulation results in the formation of the three primary germ layers-ectoderm, mesoderm, and endoderm - and in the establishment of the basic body plan of the mouse embryo (Beddington and Robertson 1999; Tam and Loebel 2007). Prior to gastrulation, at embryonic day 5.5 (E5.5), a group of visceral endoderm (VE) cells at the distal tip of the embryo differentiates into a morphologically distinct tissue termed distal VE (DVE) (Srinivas 2006). The DVE expresses characteristic molecular markers such as Hex, Leftyl, and Dkk1 and mi-

\footnotetext{
${ }^{5}$ These authors contributed equally to this work.

${ }^{6}$ Present address: LMU München, Medical Department, Schillerstraße 46, 80336 München, Germany.

Corresponding authors.

${ }^{7}$ E-MAIL rklein@neuro.mpg.de; FAX 49-89-8578-3152

${ }^{8}$ E-MAIL jegea@neuro.mpg.de; FAX 44-89-8995-0118.

Article is online at http://www.genesdev.org/cgi/doi/10.1101/gad.486708.
}

grates from the distal tip of the embryo to a more proximal region to give rise to the anterior $\mathrm{VE}(\mathrm{AVE})$, which at E6.5 positions itself above the prospective anterior epiblast (Tam and Loebel 2007). Failure of AVE cells to migrate as in the case of embryos deficient in Lim1, Otx2, or Foxa2/Hnf $3 \beta$ results in loss of anterior neural induction due to lack of AP patterning in the epiblast (Tam and Loebel 2007). Recent work has shown that the AVE (and the chick equivalent, the hypoblast) controls anterior-posterior (AP) patterning by distinct processes. For instance, the AVE restricts primitive streak (PS) formation and mesoderm induction to the posterior side of the epiblast by expressing antagonists (Cer1, Leftyl, and Dkk1) of the posteriorizing activities of Nodal and Wnts (Lu et al. 2001; Bertocchini and Stern 2002; Perea-Gomez et al. 2002; Rossant and Tam 2004). Also, the AVE has been proposed to direct epiblast movements through induction of the Wnt planar cell polarity pathway 
(Voiculescu et al. 2007). Finally, the AVE can also initiate the transient expression of neural markers (Albazerchi and Stern 2007).

Epiblast cells undergo epithelial-to-mesenchymal transition (EMT) and ingress into the PS region to give rise to mesoderm and definitive endoderm (DE). While the mesoderm migrates over a long distance to eventually give rise to the mesodermal organs, the DE intercalates into the overlying VE and gradually displaces the VE into the extraembryonic region, where it forms the endodermal component of the yolk sac (YS). EMT is characterized by the loss of cell polarity and the initiation of cell migration (Thiery and Sleeman 2006). At the molecular level, EMT is associated with FGF-induced down-regulation of the cell-cell adhesion protein E-cadherin, the breakdown of the basement membrane (BM), a thin sheet of extracellular matrix that underlie epithelia, and the up-regulation of EMT and mesendodermal genes such as Brachyury (T), Foxa2, Snail, Eomes, and FGF8 (Ciruna and Rossant 2001; Tam and Loebel 2007; Arnold et al. 2008).

The BM controls cell migration, differentiation, and cell fate during early embryogenesis. In the pregastrulation embryo, epiblast cells in contact with the BM produced by the VE polarize and differentiate into ectoderm epithelium, whereas epiblast cells that fail to contact the BM undergo apoptosis (Li et al. 2003). For EMT to occur in the PS, the formation and integrity of the BM require dynamic regulation. The BM has to break down locally to allow the separation of the newly formed mesoderm and endoderm from the remaining ectoderm (Fujiwara et al. 2007). The molecular cues that regulate BM dynamics during gastrulation are poorly characterized.

Fibronectin leucine-rich transmembrane protein 3 (FLRT3) belongs to a small subfamily (FLRT1-3) of putative type I transmembrane proteins (Lacy et al. 1999). While the functions of FLRT1 and FLRT2 are essentially unknown, Xenopus FLRT3 has been proposed to form a complex with fibroblast growth factor receptors (FGFRs) and to activate intracellular signals, such as the canonical MAPK pathway, which results in ectopic tail formation (Bottcher et al. 2004). FLRTs also promote homotypic cell sorting, independent of FGFR signaling, in mammalian cells or Xenopus embryos, possibly by acting as homophilic cell adhesion molecules (Karaulanov et al. 2006). Conversely, FLRT3 was shown to promote deadhesion of migrating animal cap cells during Xenopus gastrulation through the internalization of C-cadherin (Ogata et al. 2007). Genetic deletion of FLRT3 in the mouse resulted in ventral closure and headfold fusion defects suggesting that FLRT3 may be required for tissue fusion (Maretto et al. 2008). The observed anterior truncations in a fraction of $F L R T 3^{-/-}$embryos were suggested to result from a reduction in the number of anterior DE cells (Maretto et al. 2008) as described for mutations affecting Nodal signaling (for review, see Lu et al. 2001). Surprisingly, the phenotypes caused by loss of FLRT3 expression were not associated with defects in FGF signaling (Maretto et al. 2008).

To directly test the function of FLRT3 in vivo, we generated an independent line of $F L R T 3^{-/-}$mice. While we observed similar defects in our FLRT3 ${ }^{-/-}$mice as described before (Maretto et al. 2008), our analysis shows that FLRT3 is required in the VE but not in the DE. Moreover, we show that AVE signaling properties seem intact in the FLRT3 ${ }^{-/-}$embryos. However, the FLRT3-/AVE fails to maintain a functional BM, which leads to the loss of cell polarity and the induction of some aspects of EMT and mesoderm differentiation in adjacent epiblast cells as it normally occurs in the PS region. Our results therefore indicate that the AVE has a dual role: as signaling center to establish AP patterning as well as to promote epithelial cell intercalation movement and, as a morphogenetic regulator of BM integrity, to confine EMT and mesoderm induction to the PS.

\section{Results}

\section{Genetic ablation of FLRT3 arrests embryonic development}

We generated two different knockout alleles of FLRT3. In the FLRT3- allele, the entire coding sequence of FLRT3 was replaced by the neomycin (neo) gene; in the FLRT3 ${ }^{\text {lacZ }}$ allele, the coding sequence of FLRT3 was replaced by the lac $Z$ gene encoding $\beta$-galactosidase ( $\beta$-gal) followed by the neo cassette (Supplemental Fig. S1). Expression analysis demonstrated the absence of FLRT3 protein in FLRT3 $3^{-/-}$or FLRT3 ${ }^{\text {lacZ/lacZ }}$ embryos (Supplemental Figs. S1, S2). The $\beta$-gal activity encoded by the FLRT3 ${ }^{\text {lacZ }}$ allele faithfully reflected the pattern of FLRT3 mRNA (Supplemental Fig. S2; see also Haines et al. 2006). FLRT3 heterozygous intercrosses in three independent genetic backgrounds (129/SvEv, 129/ SvEv $\times$ C57BL/6, and 129/SvEv $\times$ CD1) (Supplemental Fig. S3) yielded $<3 \%$ viable FLRT3 ${ }^{-/-}$or FLRT3 $3^{\text {lacZ/lacZ }}$ mice at postnatal day 15 , indicating that the mutation is embryonic lethal (Supplemental Fig. S3). At midgestation, FLRT3-/- embryos suffered from a range of malformations including anterior neural tube closure defects, asymmetric development of the headfolds, and failure of embryonic turning and ventral body closure, as well as cardia bifida as described previously (Supplemental Fig. S3; Maretto et al. 2008). In most severe cases, the embryos developed outside the YS and were extremely growth-retarded (Supplemental Fig. S3).

\section{Expression of FLRT3 in VE and DE of the early embryo}

Because these malformations could originate from patterning defects and/or morphogenetic events during gastrulation, we investigated FLRT3 expression between E6.0 and E8.0. In E6.0 prestreak (PrS) stage FLRT3+/lacZ embryos, FLRT3 expression (as monitored by $\beta$-gal activity) was highest in the chorion and in the VE surrounding the FLRT3-negative epiblast (Fig. 1A-C). FLRT3 expression was uniform with respect to the fu- 


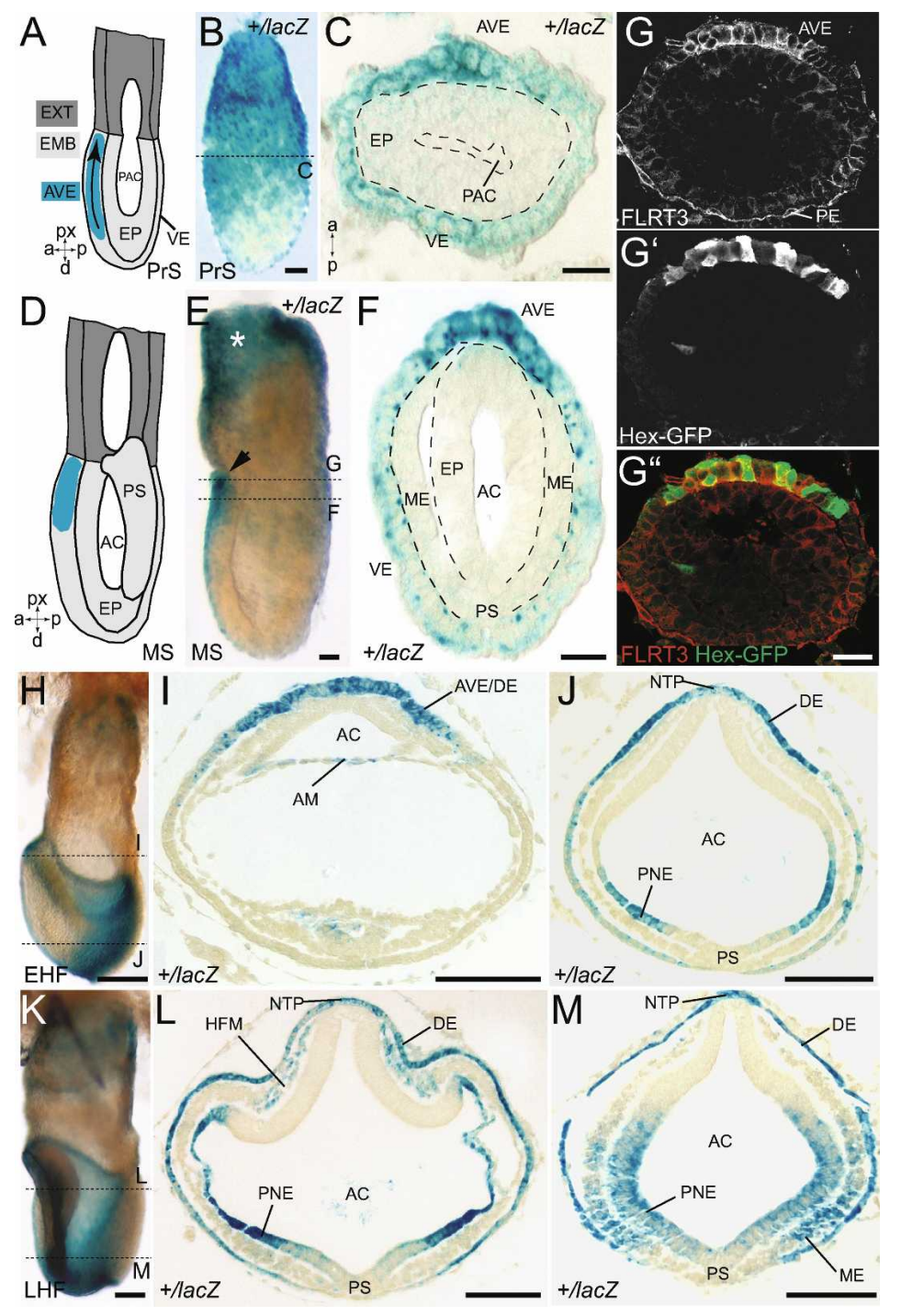

Figure 1. Expression of FLRT3 in the early mouse embryo. (A) Schema of a PrS stage (E6.0) embryo. (px) Proximal; (d) distal; (a) anterior; (p) posterior. The embryo is divided into two parts: the extraembryonic (dark gray) and the embryonic (light gray), which is subdivided in epiblast, which will give rise to the embryo, and VE. The distal-to-proximal migration of AVE cells is indicated (arrow within the blue domain). (B) XGal-stained PrS stage $F_{L R T 3^{+/ l a c Z}}$ embryo (same orientation as in $A$ ). (C) Crosssection at the position of the dotted line in $B$. Dashed lines outline the proamniotic cavity and the epiblast. Anterior to the top. $(D)$ Schema of a MS stage (E6.75-E7.0) embryo. AVE cells have arrived at the prospective anterior region of the embryo and PS formation has begun in the posterior side. (E) XGal-stained MS stage FLRT3 $3^{+/ l a c Z}$ embryo (same orientation as in $D$ ). FLRT3 expression remains high in the extraembryonic ectoderm (asterisk) and in the anterior region of the embryo (arrow). (F) Crosssection at the position of the dotted line in E. FLRT3 expression is in an anterior high to posterior low gradient exclusively in the VE. Dashed lines outline the epiblast and mesoderm. $\left(G-G^{\prime \prime}\right)$ Cross-sections at the position of the dotted line in $E$ of a MS stage embryo carrying the Hex-GFP transgene. $(G)$ Immunostaining for FLRT3. $\left(G^{\prime}\right)$ GFP fluorescence. $\left(G^{\prime \prime}\right)$ Merged image showing colocalization of FLRT3 and Hex in the anterior region of the embryo. (H-J) XGal-stained EHF stage (E7.25) and $(K-M)$ LHF stage (E7.75) FLRT3 ${ }^{+/ l a c Z}$ embryos. Positions of the crosssections $(I, J, L, M)$ are indicated by dotted lines in $H$ and $K$. FLRT3 is expressed in AVE and DE, amnion, posterior neuroectoderm, headfold, and posterior mesoderm. FLRT3 expression is absent or low in ectoderm underlying headfolds and in the PS. (AC) Amniotic cavity; (AM) amnion; (AVE) anterior visceral endoderm; (DE) definitive endoderm; (EHF) early head fold; (EMB) embryonic region; (EP) epiblast; (EXT) extraembryonic region; (HFM) headfold mesoderm; (LHF) late head fold; (ME) mesoderm; (MS) mid-streak; (NTP) notochordal plate; (PAC) proamniotic cavity; (PE) parietal endoderm; (PNE) posterior neuroectoderm; (PrS) prestreak; (PS) primitive streak; (VE) visceral endoderm. Bars: $B, C, E-G^{\prime \prime}, 25 \mu \mathrm{m} ; H-M, 100 \mu \mathrm{m}$. ture AP axis. In E6.75-E7.0 mid-streak (MS) stage embryos, FLRT3 expression was still present in the embryonic VE but had shifted into an anterior high to posterior low gradient (Fig. 1D-F) and stopped abruptly at the boundary between the embryonic and the extraembryonic part (Fig. 1E). FLRT3 expression in AVE cells was confirmed using a transgenic line that expresses enhanced green fluorescent protein (EGFP) under the control of Hex regulatory sequences (Rodriguez et al. 2001). EGFP fluorescence overlapped with FLRT3 immunoreactivity in the AVE region of the embryo (Fig. 1G-G"; see also Supplemental Fig. S2 for antibody specificity). In approximately E7.25 early headfold (EHF) stage embryos, FLRT3 expression continued in the most anterior region of the embryo, which at this stage is populated by DE cells (Srinivas 2006), and additionally appeared in the posterior neuroectoderm (Fig. 1H-J). In approximately E7.75 late headfold (LFH) stage embryos, FLRT3 continued to be expressed in DE, parts of the neuroectoderm and was induced in headfold and posterior mesoderm
(Fig. 1K-M). FLRT3 expression was conspicuously low in the PS region at all the stages analyzed (Fig. 1F,J,L,M).

\section{Rupture of the AVE in FLRT3 knockout embryos}

FLRT3-/- embryos displayed abnormalities already at the MS/EHF stage. In FLRT3 ${ }^{+/ l a c Z}$ embryos, XGal-stained VE cells formed a continuous epithelial cell layer and accumulated in a region overlaying the anterior epiblast (Fig. 2A,B). In mutant FLRT3-/lacZ embryos, XGal-positive VE cells were also found in the AVE region of the embryo; however, these embryos often showed areas completely devoid of XGal-positive cells (Fig. 2C,D). Crosssectioned embryos revealed that these XGal-negative areas lacked VE cells and represented holes through which the subjacent epiblast was exposed (Fig. 2E,F). The penetrance of this phenotype was high, as 19 out of $24 \mathrm{mu}-$ tant embryos analyzed by XGal staining showed holes in the anterior part of the embryo (see also Fig. 5, below). To obtain a three-dimensional impression of this pheno- 
Figure 2. Rupture of the AVE in $F L R T 3^{-/-}$embryos. $(A-G)$ XGal-stained MS stage embryos. (A) FLRT3+/lacZ control embryo. Asterisk indicates the AVE. $(B)$ Crosssection at the position indicated by the dotted line in $A$ (anterior to the top). (C,D) FLRT3-/lacZ embryo displaying an area free of XGal-positive cells in the anterior, most proximal part of the embryo, where $\beta$-gal activity is highest (arrows). $(E-G)$ Sections of FLRT3-/lacZ embryos. Asterisks indicate the holes in the VE. Dashed line in $B$, and $E-G$ outlines the epiblast. $(H, I)$ Sections of MS stage embryos, immunostained for Sox2 (red) and counterstained with DAPI (blue). In mutant embryos, Sox2-positive cells are facing the outside (arrows) where the endoderm has ruptured. Dashed lines outline the epiblast. (J-Q) SEM images of EHF embryos. The circumferential groove is indicated by a dashed line. $(J, L, M)$ Frontal view of a control embryo $(J)$ and higher magnifications of the VE $(M)$ and $\mathrm{DE}(L)$ of the regions indicated in $J .(K, N, O)$ Frontal views of $F L R T 3^{-/-}$embryos with a shallow hole $(K)$, a deep hole $(N)$, or two holes $(O)$ (arrowheads). $(P, Q)$ Higher magnification of the areas in $N$ and $O$ showing a new compact cell type with a smooth surface and a recognizable profile (arrowheads). Asterisks indicate DE cells. Dashed line in $Q$ outlines an area of DE cells. (EP) Epiblast; (VE) visceral endoderm. Bars: $A-C, E-I, 25 \mu \mathrm{m} ; J, K, N, O, 20 \mu \mathrm{m}$.

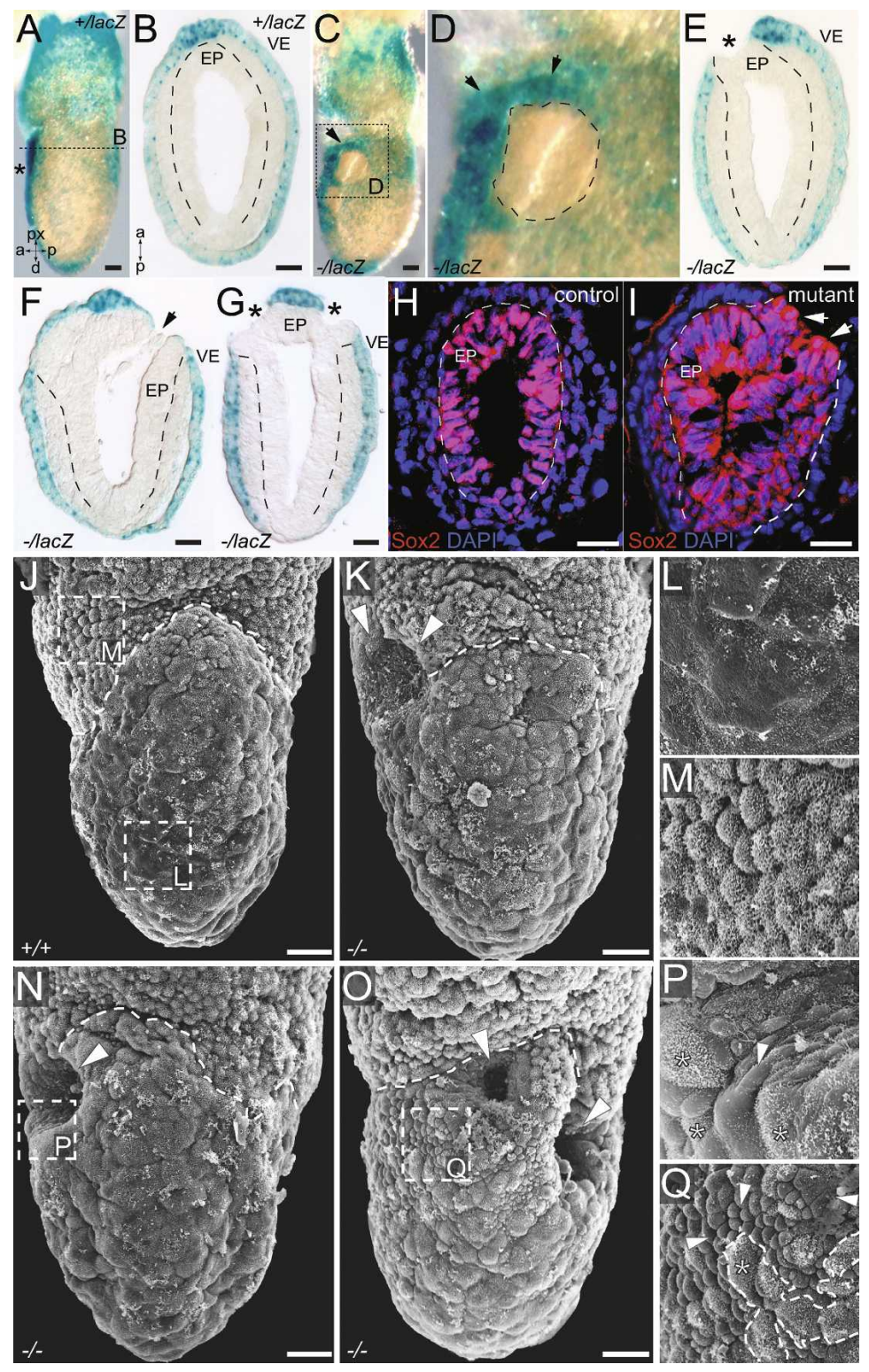

type, we analyzed EHF stage $F L R T 3^{-/-}$embryos by scanning electron microscopy (SEM). In control embryos, extraembryonic VE cells could be distinguished by their dense coat of microvilli (Fig. 2J,M; Ishikawa et al. 1986). In contrast, DE showed no clear outlines of cells and fewer microvilli on their surface (Fig. 2J,L). The boundary between the VE and the DE could be easily distinguished by the circumferential groove (Tam and Meier 1982). In FLRT3-/- embryos, one or rarely two holes formed at this boundary. These holes were shallow (Fig. $2 \mathrm{E}, \mathrm{K}$ ) and sometimes deep (Fig. 2F,G,N,O). They uncovered a new compact cell type with a smooth surface and a recognizable profile that appeared to be epiblast cells (Fig. 2P,Q). Immunostaining with Sox2, a neuronal marker specific for epiblast cells in MS stage embryos (Wood and Episkopou 1999), confirmed that epiblast cells have reached the surface in mutant embryos where the surrounding endoderm had formed a hole (Fig. 2H,I).
These results demonstrate that FLRT3 is required to maintain the integrity of the AVE of MS stage embryos.

\section{FLRT3 is required for morphogenesis, but not patterning of the embryo}

We next investigated if the rupture of the AVE was linked to the anterior head formation defects observed in older embryos. As discussed above, a large percentage $(79 \%)$ of $F L R T 3^{-/-}$embryos displayed unilateral holes in the anterior endoderm (Fig. 3A-E). A similar high percentage of E8.5-E9.5 embryos suffered from unilateral headfold atrophies or even more severe malformations (Fig. 3G; Supplemental Fig. S3). It appeared as if one of the headfolds had stopped development, while the other continued developing normally. Holes in the VE and atrophied headfolds appeared randomly on either the left or the right side. To begin investigating if these two 

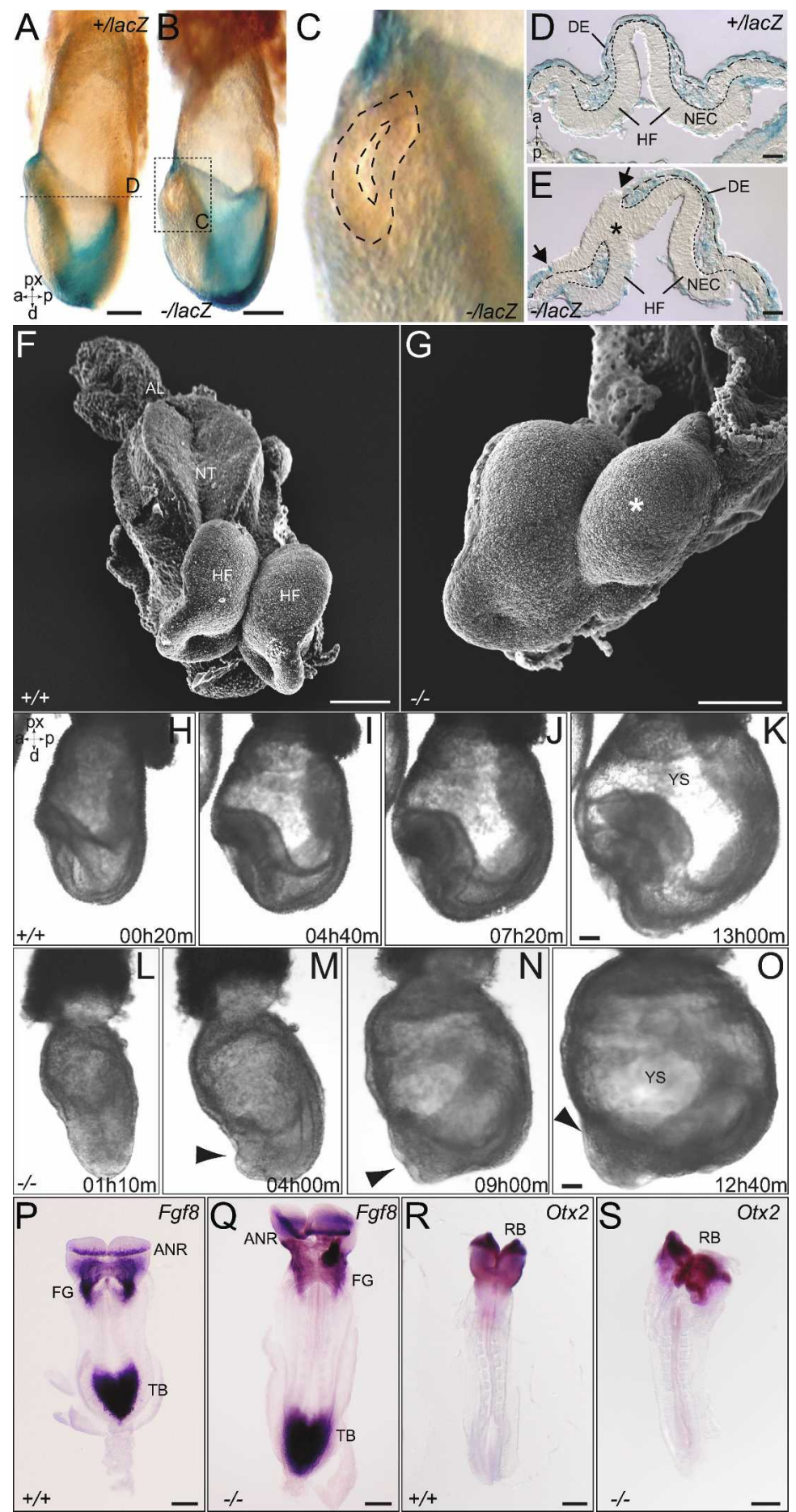
-
Figure 3. Head formation defects in $F L R T 3^{-/-}$embryos. $(A-E)$ XGal-stained EHF stage $F L R T 3^{+/ 1 a c Z}$ embryo $(A, D)$ and $F L R T 3^{-/ l a c Z}$ embryo $(B, C, E)$. $(D, E)$ Cross-sections at the position of the dotted line in $A$ and $B$ (only the anterior part is shown, anterior to the top). $(A, D)$ The $F L R T 3^{+/ \text {lacz }}$ embryo has a normal appearance with an intact and continuous DE. The $F L R T 3^{-/ l a c Z}$ embryo displays a hole in the anterior region $(B$, dashed line in $C)$ through which the neuroectoderm protrudes and replaces the $\mathrm{DE}(E$, arrows), forming a continuous epithelial layer with the head folds (HF) (E, asterisk). Dotted and dashed lines in $D$ and $E$ outline the neuroectoderm and the DE, respectively. $(F, G)$ SEM images of E8.0 control $(F)$ and $F L R T 3^{-/-}$embryos $(G)$; asterisk indicates the smaller headfold in the $\mathrm{FLRT3}^{-{ }^{--}}$mutant embryo. $(\mathrm{H}-\mathrm{O})$ Individual frames of in vitro cultured EHF/LHF stage embryos imaged by time-lapse video microscopy (time is indicated, hours:minutes). ( $H-K)$ Control embryo developing inside the YS. $(L-O)$ The anterior portion of the $F L R T 3^{-/-}$embryo including the neuroectoderm breaches through the YS (arrowhead). $(P-S)$ In situ hybridization of control $(P, R)$ and $F L R T 3^{-/-}$embryos $(Q, S)$ at E8.0-E8.5 for Fgf8 or Otx2. (AL) Allantois; (ANR) anterior neural ridge; (DE) definitive endoderm; (FG) foregut; $(\mathrm{HF})$ headfold; (NEC) neuroectoderm; (NT) neural tube; $(\mathrm{RB})$ rostral brain; (TB) tailbud; (YS) yolk sac. Bars: $A, B, 100 \mu \mathrm{m}$; $D, E, 25 \mu \mathrm{m} ; F, G, 100 \mu \mathrm{m} ; K, O, 100 \mu \mathrm{m} ; P-S, 200 \mu \mathrm{m}$. events may be causally linked, we imaged EHF stage embryos in culture (Srinivas et al. 2004). Embryos were dissected and set up at the EHF stage, and phase-contrast images were captured every 10 min over a period of $15 \mathrm{~h}$. During this period, control embryos gained significantly in size (Fig. 3H-K; Supplemental Movie 1). The growth of the headfolds caused the YS to bulge outward, suggesting that they exerted mechanical stress on the YS. In all the imaged control embryos, the YS remained intact, and the headfolds were contained inside $(n=17) . F^{2} R^{-1-}$ embryos showed similar growth during the culture period. However, the YS failed to resist the mechanical force of the growing headfolds, and the anterior part of the embryo broke through it $(n=3)$ (Fig. 3L-O; Supplemental Movie 2). These results suggest that FLRT3 functions to maintain a proper VE epithelium that could resist the physical stress executed by the growing embryo, and they further raise the possibility that the asymmetric 

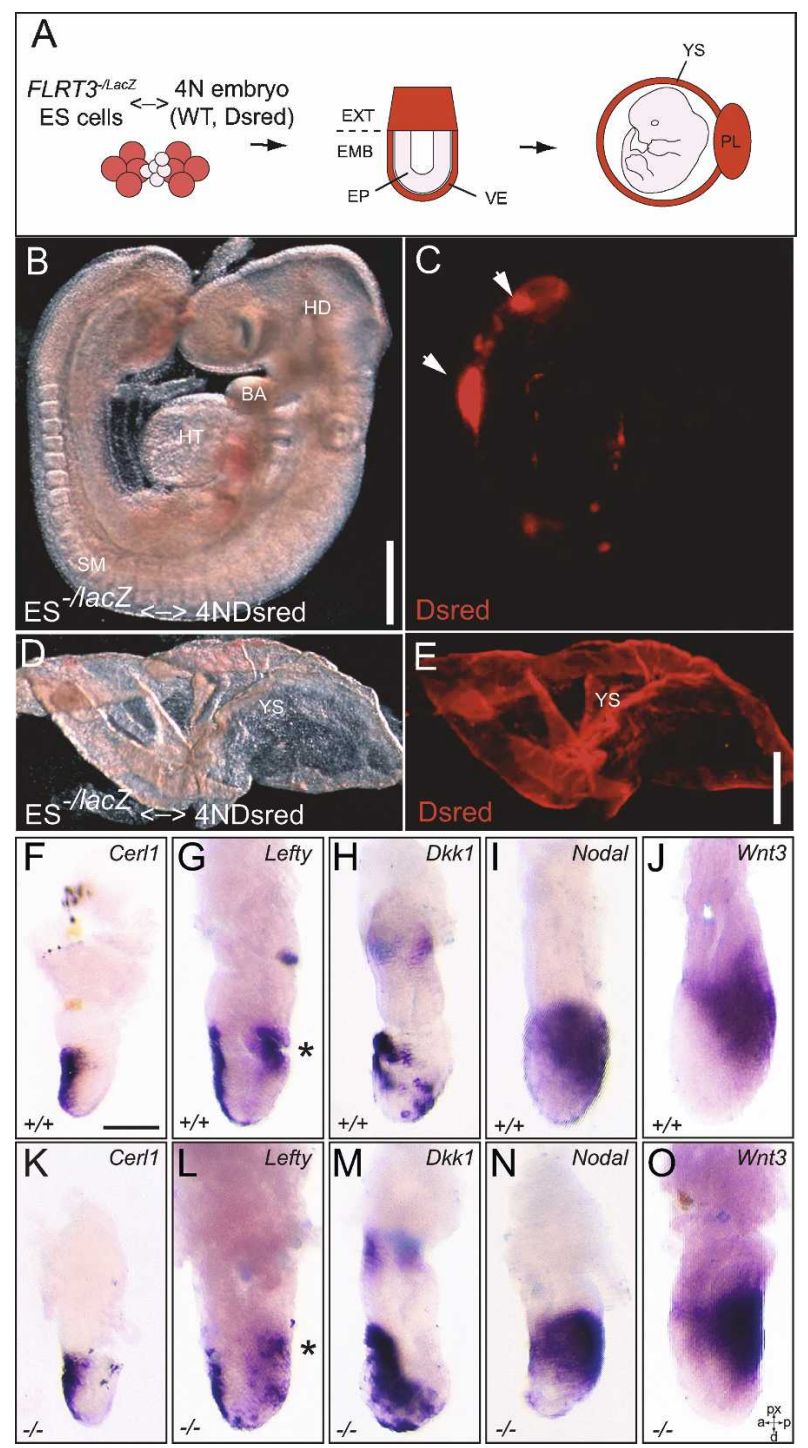

Figure 4. Tetraploid rescue of the FLRT3-/- phenotype. (A) Schema of the generation of a completely ES cell-derived embryo by aggregation of $F L R T 3^{-/ 1 a c Z}$ ES cells with wild-type DsRed labeled tetraploid (4N) embryos. (B) Representative E9.5 embryo obtained by the aggregation protocol depicted in $A .(C)$ Fluorescence image of the embryo shown in $B$. The low contribution of the $4 \mathrm{~N}$ cells to the embryo is restricted to the tail region (arrows). $(D, E)$ Bright-field $(D)$ and fluorescent $(E)$ images of the DsRed-expressing YS of the embryo shown in $B$. $(F-O)$ Representative in situ hybridization images of early-to-MS stage embryos for Cer1 $(F, K)$, Lefty1 $(G, L), D k k 1(H, M)$, Nodal $(I, N)$, and Wnt3 $(J, O)$ of control and $F L R T 3^{-1-}$ embryos (three or more embryos per genotype and probe). Lefty1 expression was detected with a mixed Lefty1/Lefty2 probe. At this stage, Lefty2 expression is restricted to the PS (asterisks) and does not overlap with Lefty1. (BA) Branchial arch; (EMB) embryonic region; (EP) epiblast; (EXT) extraembryonic region; (HD) head; (HT) heart; (PL) placenta; (VE) visceral endoderm; (WT) wild-type; (YS) yolk sac. Bars: $B-E, 500$ $\mu \mathrm{m} ; \mathrm{F}-\mathrm{O}, 100 \mu \mathrm{m}$.

headfolds seen in older embryos are an indirect consequence of a partial protrusion of the head through the YS. We next asked if $F L R T 3^{-/-}$embryos had anterior pattern- ing defects. We found that $F L R T 3^{-/-}$embryos displayed the expected pattern of FGF8 expression in the anterior neural ridge, foregut, and tailbud (Crossley and Martin 1995) and Otx2 expression in the rostral brain (Fig. 3P-S; Simeone et al. 1993). Together with data of other markers for mid- and hindbrain, midline structures, and somite mesoderm (Supplemental Fig. S4), the findings suggest that patterning was unaffected in $F L R T 3^{-1-} \mathrm{em}-$ bryos.

\section{Rescue of the FLRT3 $^{-/}$phenotypes by tetraploid chimera experiments}

FLRT3 is expressed in both AVE and DE. In order to understand if FLRT3 was required in AVE, DE, or both, we took advantage of the developmental bias whereby embryonic stem (ES) cells that were aggregated with a normal diploid embryo give rise to structures of the fetus, but do not contribute to extraembryonic endoderm or trophectoderm (Supplemental Fig. S5; Tam and Rossant 2003). In cases in which FLRT3-/lacZ ES cells contributed extensively to the embryo proper, including the $\mathrm{DE}$, we did not observe the high incidence of anterior defects observed in FLRT3 ${ }^{-/-}$embryos (19 out of 25 highly chimeric embryos [E6.5-E10.5] derived from FLRT3-/lacZ ES cells were morphologically normal) (Supplemental Fig. S5). We also generated completely ES cell-derived embryos by aggregating FLRT3-/lacZ ES cells with wild-type tetraploid embryos. In such an experiment, the descendents of the tetraploid cells contribute exclusively to extraembryonic structures, such as VE, YS, and the placenta (Fig. 4A; Tam and Rossant 2003). Importantly, all $(n=5)$ of the $F L R T 3^{-/ 1 a c Z}$ embryos, dissected between E8.5 and E9.5, developed normally, and none showed anterior head or ventral body closure defects indicating a requirement for FLRT3 in VE, but not DE (Fig. 4B-E). These results further suggest that the head formation phenotypes observed in midgestation $F L R T 3^{-/-}$embryos are indirectly caused by rupture of the VE and are not a consequence of lack of FLRT3 in embryonic tissues. We next asked if a failure of the signaling function of the AVE was responsible for the phenotype of $\mathrm{FLRT}^{-/-}$embryos. To this end, we analyzed the expression pattern of the Nodal inhibitors Cer1 and Lefty1 and of the Wnt inhibitor Dkk1 by in situ hybridization in early to MS stage embryos and found that their levels and spatial distribution was unaffected in FLRT3 ${ }^{-/-}$embryos (Fig. 4F-H,K-M, three or more mutant embryos per genotype and probe; see also Maretto et al. 2008). Moreover, we were unable to detect an expansion of the expression domains of Wnt3 or Nodal from posterior to anterior region (Fig. $4 \mathrm{I}, \mathrm{J}, \mathrm{N}-\mathrm{O}$, three or more mutant embryos per genotype and probe). These results suggest that AP patterning is normal and the signaling properties of the AVE remain intact in FLRT3 ${ }^{-/-}$embryos.

\section{FLRT3 is not required for proliferation, survival,} or migration in early development

Since one of the proposed functions of FLRT3 is to promote FGF receptor signaling (Bottcher et al. 2004), the 
lack of FLRT3 may reduce cell proliferation in the VE. Using phosphorylated histone $\mathrm{H} 3$ as a marker for mitotic cells (Cho et al. 2003), we found that the mitotic index in the FLRT3-/- embryos was indistinguishable from controls (Supplemental Fig. S6). Since the growth rate of a tissue is influenced by the rate of cell death within the tissue, we immunostained embryos for active caspase-3, a marker for apoptotic cells, and found very few apoptotic cells in control and FLRT3 ${ }^{-/-}$embryos (Supplemental Fig. S6; data not shown). Finally, we asked if FLRT3-/VE cells failed to respond to directional cues, as it was reported for Lefty1 or Cer1 knockouts (Yamamoto et al. 2004; Kimura-Yoshida et al. 2005; Srinivas 2006). Live imaging of mutant embryos carrying the Hex-EGFP transgene showed that the EGFP-positive cells migrated properly toward the anterior part of the embryo (Supplemental Fig. S6). Together with the normal expression pattern of Cer1, Lefty1, and Dkk1 (Fig. 4), we concluded that distal-to-proximal movement of the AVE was normal in FLRT3 ${ }^{-1-}$ embryos. In summary, these results suggest that $F L R T 3^{-/-}$cells display normal cell proliferation, survival, and migration.
FLRT3 $^{-/-}$embryos display a ruptured BM that precedes the disruption of the $V E$

Previous work has shown that FGFR signaling is important for the expression of laminin111 (LN111) and collagen IV, which control BM assembly during embryoid body (EB) differentiation (Li et al. 2001). To investigate if loss of FLRT3 affected the production of BM components, we immunostained MS stage embryos for LN111, collagen IV, and perlecan. Control embryos displayed a continuous BM between the anterior endoderm and the subjacent epiblast (Fig. 5A-D). In sharp contrast, all FLRT3-/- littermates displayed a disrupted BM in the anterior-lateral aspect of the embryo (Fig. 5E-J). Often, BM components were found ectopically located in the amniotic cavity and scattered in the epiblast (Fig. 5F-H). FLRT3 $^{-/-}$embryos were categorized according to the position of BM breakdown (sector i, anterior; sector ii, lateral, up to the tips of the mesodermal wings; sector iii, posterior to the tips of the mesodermal wings). The location of the disrupted BM was highly biased toward the anterior-lateral region of the embryo (86\% were in sector
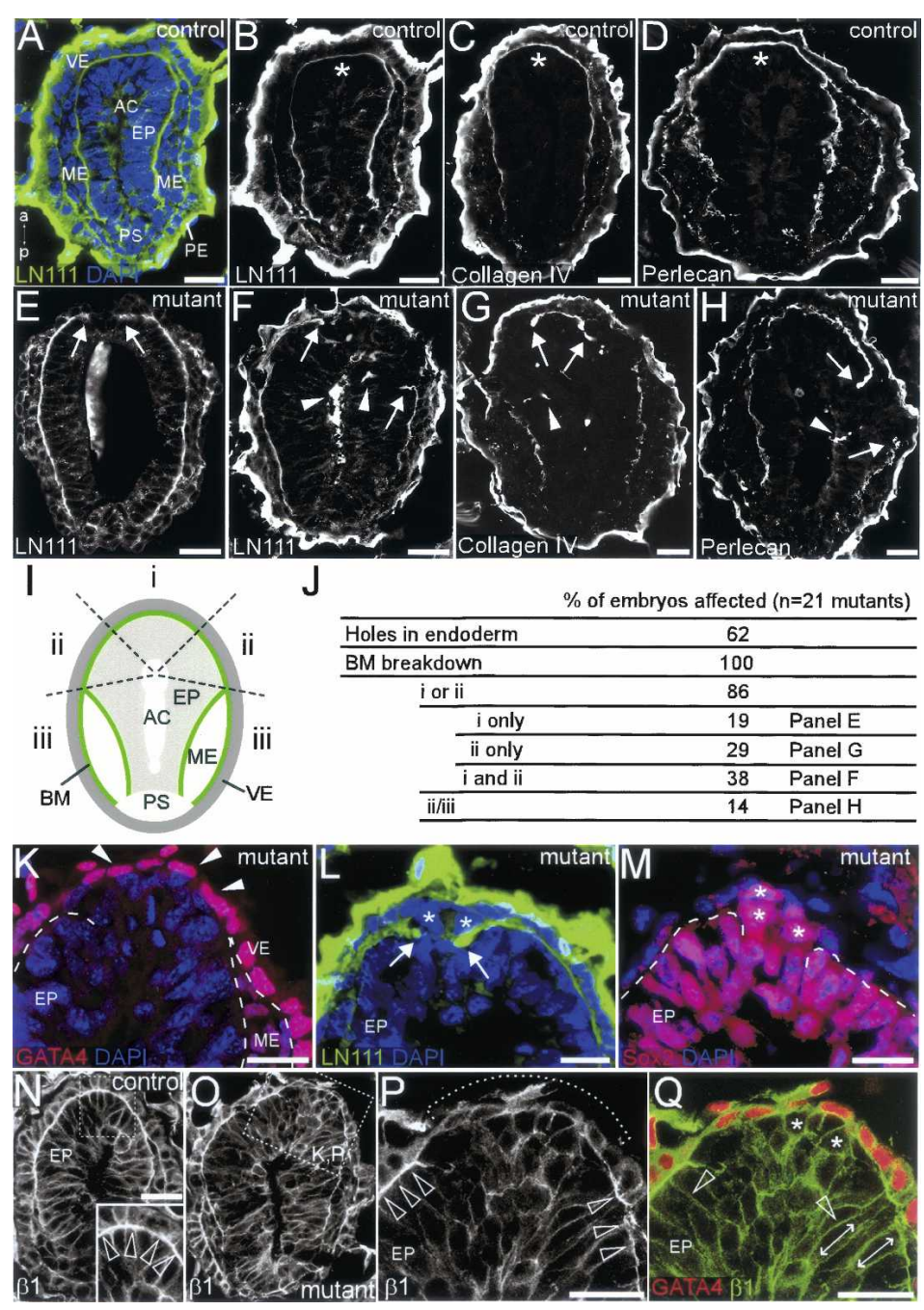

Figure 5. FLRT3-/- embryos display a failure of BM organization that precedes the disruption of the VE. $(A-$ $H$ ) Confocal images of sectioned MS stage control $(A-D)$ or mutant $(E-H)$ embryos immunostained for LN111, collagen IV, or perlecan (section $A$ is counterstained with DAPI). Anterior to the top. $(B-D)$ In controls, the $\mathrm{BM}$ forms a continuous thin layer between VE and epiblast in the anterior region (asterisk). (E-H) FLRT3-/embryos showed a ruptured BM in the anterior portion of the embryo (arrows). BM components were often ectopically localized in the epiblast and in the amniotic cavity (arrowheads). (I) Schema of a section divided in sector i (anterior), sector ii (lateral, up to the tips of the mesodermal wings), and sector iii (posterior to the tips of the mesodermal wings). (J) Summary of the incidence of the BM breakdown in the regions depicted in $I$. Examples for each category are indicated. $(K-M)$ BM breakdown happens before holes appear in the VE. $(K)$ Anterior portion of the $\mathrm{FLRT3}^{-/-}$section shown in $O$ and immunostained for GATA4 showing an intact endoderm (arrowheads) in the region where BM is disrupted (dashed line). (L) Anterior portion of a $F L R T 3^{-/-}$section immunostained for LN111 and counterstained with DAPI showing ectopic cells (asterisks) that migrated through the hole in the BM (arrows). ( $M$ ) Adjacent section to $L$ showing that these ectopic cells are of epiblast origin (Sox2+, asterisks). The dashed line outlines the BM. $(N)$ Section of a control embryo immunostained for $\beta$ lintegrin. VE and epiblast cells display polarized, basal, $\beta$ lintegrin expression (open arrowheads). (O-Q) Section of a FLRT3-/- embryo immunostained for $\beta$ lintegrin. VE and epiblast cells in the region where the $\mathrm{BM}$ was disrupted (dotted line in $P$ ) showed even distribution of $\beta 1$ integrin (asterisks in $Q)$. $(P, Q)$ Adjacent cells in contact with well-formed BM are polarized (open arrowheads and double-headed arrows). (Q) GATA4 immunostaining showing that the endodermal layer appears largely intact. (AC) amniotic cavity; (EP) epiblast; (ME) mesoderm; (PE) parietal endoderm; (VE) visceral endoderm. Bars: $A-H, N, O, 25 \mu \mathrm{m} ; K-M, P, Q, 20 \mu \mathrm{m}$. 
i or ii) (Fig. 5J). At this developmental stage, many of the $\mathrm{FLRT3}^{-/-}$embryos showed no holes and an intact VE, as shown by the endodermal marker GATA4 (Fig. 5J,K). Sox2-positive epiblast cells accumulated between the $\mathrm{BM}$ and the overlaying VE (Fig. 5L,M). Cells in the vicinity of BM breakdown lost their polarized expression of $\beta$ lintegrin and changed from a columnar shape to a rounded phenotype, which altogether is suggestive of EMT (see below) (Fig. 5N-Q). These results argue that the primary defect in FLRT3 ${ }^{-/-}$embryos is the breakdown of the BM in the anterior region of the embryo with the consequence that the endodermal cell layer becomes fragile and epiblast cells ingress.

\section{FLRT3 $^{-/-}$EBs display an abnormal morphology} and $a$ disorganized $B M$

To further investigate endoderm differentiation and BM formation, we generated EBs from control and $\mathrm{FLRT}^{-1}$ ES cells (Montanez et al. 2007). FLRT3 expression was induced during EB differentiation and was restricted to the primitive endoderm (Fig. 6A,B,D). Furthermore, FLRT3 $^{-/-}$EBs expressed endoderm markers such as GATA4 and $\alpha$-fetoprotein (AFP) and expressed and secreted (data not shown) BM components such as LN111 (Fig. 6A-D). Reminiscent of the in vivo situation, however, $\sim 40 \%$ of FLRT3 $^{-/-}$EBs formed amorphous protrusions with absence of a BM where the protrusion originated (Fig. 6E-J). Accordingly, immunostaining for LN $\alpha 1$-chain showed that a high percentage of $F L R T 3^{-/-}$ EBs $(\sim 65 \%)$ displayed a scattered and highly disorganized pattern (Fig. 6K-O). These results support the in vivo observations and suggest that FLRT3 is required to maintain and/or stabilize the BM between VE and epiblast.

BM breakdown in $\mathrm{FLRT}^{-/-}$embryos induces EMT and mesodermal gene expression in the anterior epiblast

Breakdown of the BM is an important event for EMT during gastrulation (Viebahn et al. 1995). Recent work
Figure 6. $F L R T 3^{-/-}$EBs display abnormal morphology and disorganized BM. $(A, B)$ Section through a control EB, cultured for $7 \mathrm{~d}$ in vitro (DIV), immunostained for FLRT3 and LN $\alpha 1$. FLRT3 is expressed in the basolateral compartment of the VE. The presence of a thin layer of BM between VE and epiblast indicates that the EB is well differentiated. $(C)$ Quantification of unstained EBs $(6$ $\mathrm{DIV})$ revealed that $F L R T 3^{-/-}$EBs form visible BM structures to the same extent as control EBs (two independent ES cell clones per genotype; $\mathrm{ES}^{+/+}$, clone \#1, $n=209$; clone \#2, $n=209$; $\mathrm{ES}^{-/-}$, clone \#1, $n=207$; clone \#2, $n=219$ ). (n.s.) Nonsignificant differences (ANOVA). All following experiments were performed with clone \#1 of each genotype. $(D)$ Western blot analysis of ES cell and EB protein extracts for LN111 $(\alpha, \beta$, and $\gamma$ chains), the endodermal markers GATA4 and AFP, FLRT3, and tubulin. $(E-H)$ Representative examples of EBs. $(E)$ The $\mathrm{EB}^{+/+}$is round and has a continuous BM. $(F-H)$ The $\mathrm{EB}^{-/-}$displays a discontinuous BM (arrowheads) and amorphous protrusions (asterisks). (I) Quantification of the proportion of EBs that formed protrusions. Data of five independent experiments, $>300$ EBs per genotype; $\left(^{\star \star \star}\right) P<0.01$; Student's $t$-test. (J) Shape factor analysis of the morphology of EBs. Data of three independent experiments, $>350$ EBs per genotype; $\left(^{\star \star \star}\right) P<0.01 ;\left(^{\star \star}\right) P<0.05$; Student's $t$-test. Data are plotted as the cumulative percentage. $(K-$ $N)$ Representative sections through EBs immunostained for $\mathrm{LN} \alpha 1$ and counterstained with DAPI. In the $\mathrm{EB}^{-/-}$, $\mathrm{LN} \alpha 1$ staining is scattered and discontinuous (asterisks). (O) Proportion of the EBs displaying a disorganized BM (data of two independent experiments, $\mathrm{EB}^{+/+}, n=93 ; \mathrm{EB}^{-/-}$, $\left.n=52 ;{ }^{\star \star}{ }^{\star}\right] P<0.05$; Student's $t$-test). (CAV) cavity; (EB) embryoid body; (EP) epiblast; (VE) visceral endoderm. Bars, $50 \mu \mathrm{m}$.

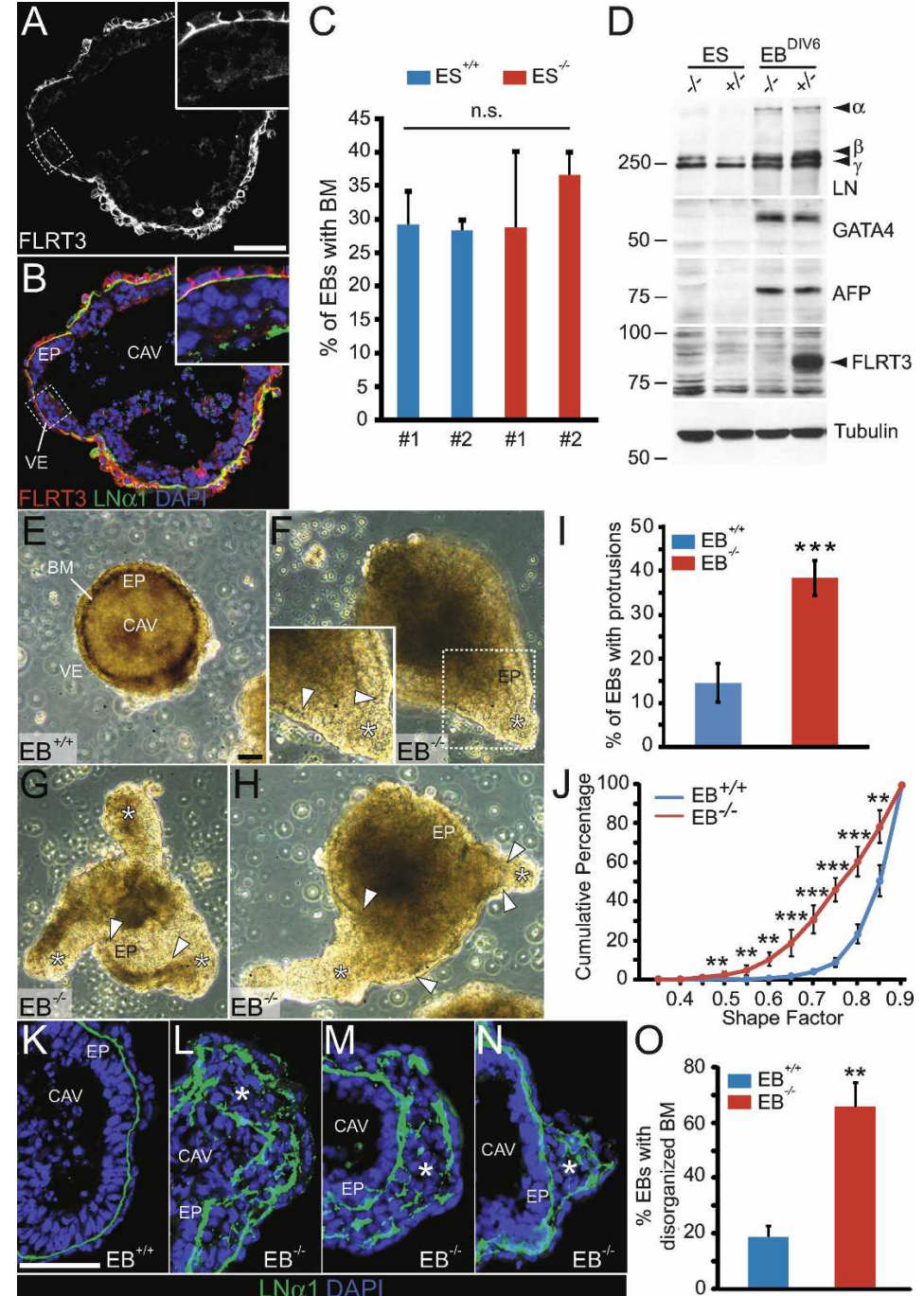


had shown that the BM prevents EMT and precocious differentiation of epiblast into mesoderm (Fujiwara et al. 2007). Interestingly, we detected ectopic $T$ expression in the heads of E8.5 FLRT3 ${ }^{-/-}$embryos (Fig. 7A,B), raising the possibility that BM breakdown triggers the ingression of the anterior epiblast cells and the acquisition of a mesodermal cell fate. To investigate this hypothesis, we performed in situ hybridization for $T$ on FLRT3-/- EHF stage embryos. The most distal portion of the ectopic outgrowth of neuroectodermal tissue in $\mathrm{FLRT3}^{-/-}$embryos showed strong $T$ expression (six out of eight embryos with endoderm disruption, from a total of $10 \mathrm{em}$ bryos) (Fig. 7D,E). This suggested that only the cells confronted with the ruptured BM received signals that induced $T$ expression. In support of the hypothesis that these cells adopt a mesodermal cell fate, we also detected expression of Fgf8 (four out of eight embryos) and Eomes (two out of two embryos) in the ectopic neuroectoderm

Figure 7. Anterior BM breakdown in $\mathrm{FLRT3}^{-1-} \mathrm{em}$ bryos induces EMT and mesodermal cell fate. $(A-K)$ In situ hybridizations on E8.5 $(A, B)$ and EHF/LHF $(C-K)$ embryos for $T(A-E), F g f 8(F, G)$, or Eomes $(H-K) \cdot(A, B)$ Bilateral domains of ectopic $T$ expression in a $F L R T 3^{-/-}$ embryo (arrows in $B$ ). (C) Section of a control embryo with normal expression pattern of $T$ in PS and prechordal plate (arrowheads). (D,E) Sections of FLRT3-/embryos with unilateral $(D)$ or bilateral $(E)$ outgrowth of neuroectoderm and ectopic $T$ expression (asterisks). ( $F)$ Expression of $F g f 8$ in a control embryo is restricted to the PS and headfold mesoderm (arrowheads).(G) Section of a FLRT3 ${ }^{-/-}$embryo with unilateral outgrowth of neuroectoderm and ectopic Fgf8 expression (asterisk). (H,I) Representative whole-mount in situ hybridization pictures for Eomes. $(J, K)$ Sections of $F L R T 3^{-/-}$embryos showing unilateral $(J)$ or bilateral $(K)$ outgrowth of neuroectoderm and ectopic Eomes expression (asterisks). Dashed lines outline the protruding neuroectoderm. $(L-P)$ Confocal images of sectioned MS stage embryos immunostained for $T(L-N)$, Sox2 $(N)$, and $\beta$ lintegrin $(O, P) .(L)$ Normal $T$ expression is limited to the PS and mesodermal cells. In the FLRT3 ${ }^{-/-}$embryo, a group of cells in the anterior epiblast ectopically expresses $\mathrm{T}$ (arrow in $M$ ) together with Sox2 (arrowheads in $N$ ). Asterisk in $N$ indicates a T-positive, Sox2-negative cell, presumably belonging to the mesoderm. (O) Polarized, basal, $\beta$ lintegrin expression in VE and epiblast cells (open arrows). Double-headed arrow indicates the elongated shape of the epiblast nucleus. $(P)$ Same section as $N$ immunostained for $\beta$ lintegrin and counterstained with DAPI. Ectopic T-positive cells in the $F L R T 3^{-/-}$embryo are round, (asterisks) and display an even distribution of $\beta$ lintegrin (open arrowheads). (AC) Amniotic cavity; (ME) mesoderm; (NEC) neuroectoderm; (NC) notochord; (PS) primitive streak; (TB) tailbud. Bars: $A, B$, $200 \mu \mathrm{m} ; C-G, J-P, 25 \mu \mathrm{m} ; H, I, 100 \mu \mathrm{m}$.

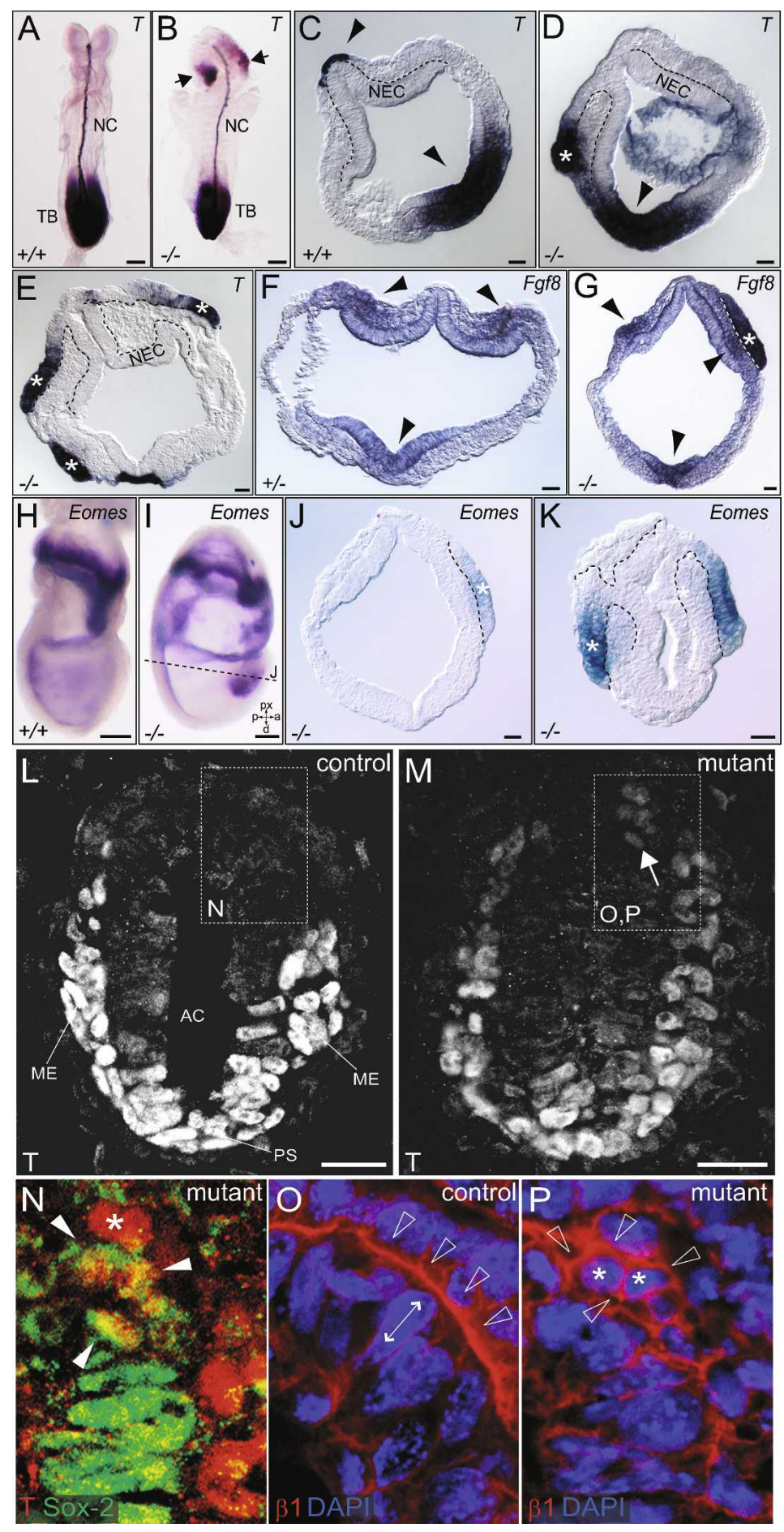


of $\mathrm{FLRT3}^{-/-}$embryos (Fig. 7F-K). However, we did not detect expression of Snai1 (data not shown), indicating that these ectopic cells acquired some, but not all, features of EMT. At earlier developmental stages, we found small groups of cells expressing $T$ protein in the anterior portion of $\mathrm{FLRT3}^{-/}$embryos (Fig. 7L-N). These cells were round and displayed an even distribution of $\beta$ lintegrin, suggesting that they had lost polarity (Fig. 7, cf. $\mathrm{O}$ and $\mathrm{P}$ ). They were part of the epiblast as shown by Sox2 staining (Fig. 7N). These results suggest that the function of FLRT3 in the anterior embryo is to maintain the BM, thereby suppressing ingression of anterior epiblast cells and differentiation of epiblast cells toward mesoderm.

\section{FLRT3 function in the AVE is independent of FGFR signaling and E-cadherin internalization}

We next asked if the function of FLRT3 in VE was related to FGFR signaling (Bottcher et al. 2004) and/or E-cadherin internalization (Karaulanov et al. 2006; Ogata et al. 2007). We found FGFR2 expression in the anterior endoderm of MS stage embryos (Supplemental Fig. S7) and interaction of FLRT3 with FGFR2 in heterologous cells (Supplemental Fig. S7). However, control and FLRT3-/GATA4-positive endodermal cells stimulated with FGF8 or FGF2 showed similar phosphorylation levels of ERK/ MAPK (Fig. 8A; Supplemental Fig. S7) and Akt (data not shown), suggesting that mouse extraembryonic endoder-
Figure 8. FLRT3 in AVE cells operates through a novel mechanism independent of FGFR signaling and E-cadherin internalization. $(A)$ Control and $F_{L R 3^{-/-}}$endodermal cells were stimulated with $100 \mathrm{ng} / \mathrm{mL}$ FGF8 for the indicated time points, then lysed and processed to visualize phosphorylated (P-)ERK/MAPK, total ERK/MAPK, GATA4, FLRT3, and tubulin (NS, nonstimulated). Molecular weight markers (in kilodaltons, $\mathrm{kDa}$ ) are indicated on the left. (B) HeLa cells were transiently transfected with vector alone (MOCK) or with plasmids encoding EGFP or FLRT3 ${ }^{\text {FLAG }}$. Cells were then stimulated with the indicated concentrations of FGF2 for $10 \mathrm{~min}$, lysed, and processed to visualize P-ERK/MAPK, total ERK/MAPK, FLRT3, EGFP, and tubulin (NS, nonstimulated). $(C-G)$ Confocal images of sectioned MS stage embryos immunostained for E-cadherin and FLRT3. Anterior to the top. $(E)$ Merge of $C$ and $D$. $(F)$ Higher magnification of dotted box in $C$. Dashed lines outline the epiblast and the VE. $(G)$ Mutant AVE cells maintain high levels of E-cadherin (arrowheads). The BM is outlined by dotted lines and the BM rupture by arrows. $(H, I)$ Bright field images of cell aggregates grown in suspension of MOCK-transfected $(H)$, or FLRT3-transfected HEK293 cells $(I)$. $(J)$ Quantification of the aggregate sizes of a representative experiment performed in quadruplicate; $\left({ }^{\star \star \star}\right) P<0.01$; Student's $t$-test. $(K, L)$ Model for the novel morphogenetic role of the AVE. $(K)$ In control MS stage embryos, the AVE produces and maintains the BM, which keeps the subjacent epiblast cells in a polarized state and prevents them from undergoing EMT and mesoderm differentiation. This morphogenetic function requires FLRT3. (L) In $F L R T 3^{-/-}$embryos, the BM breaks down locally and subjacent epiblast cells undergo some aspects of EMT and mesoderm differentiation as shown by cell rounding and expression of EMT and mesodermal markers (i.e., T). Either as an indirect consequence of BM breakdown or because of loss of FLRT3-mediated adhesion, the AVE breaks, and T-positive cells surface.

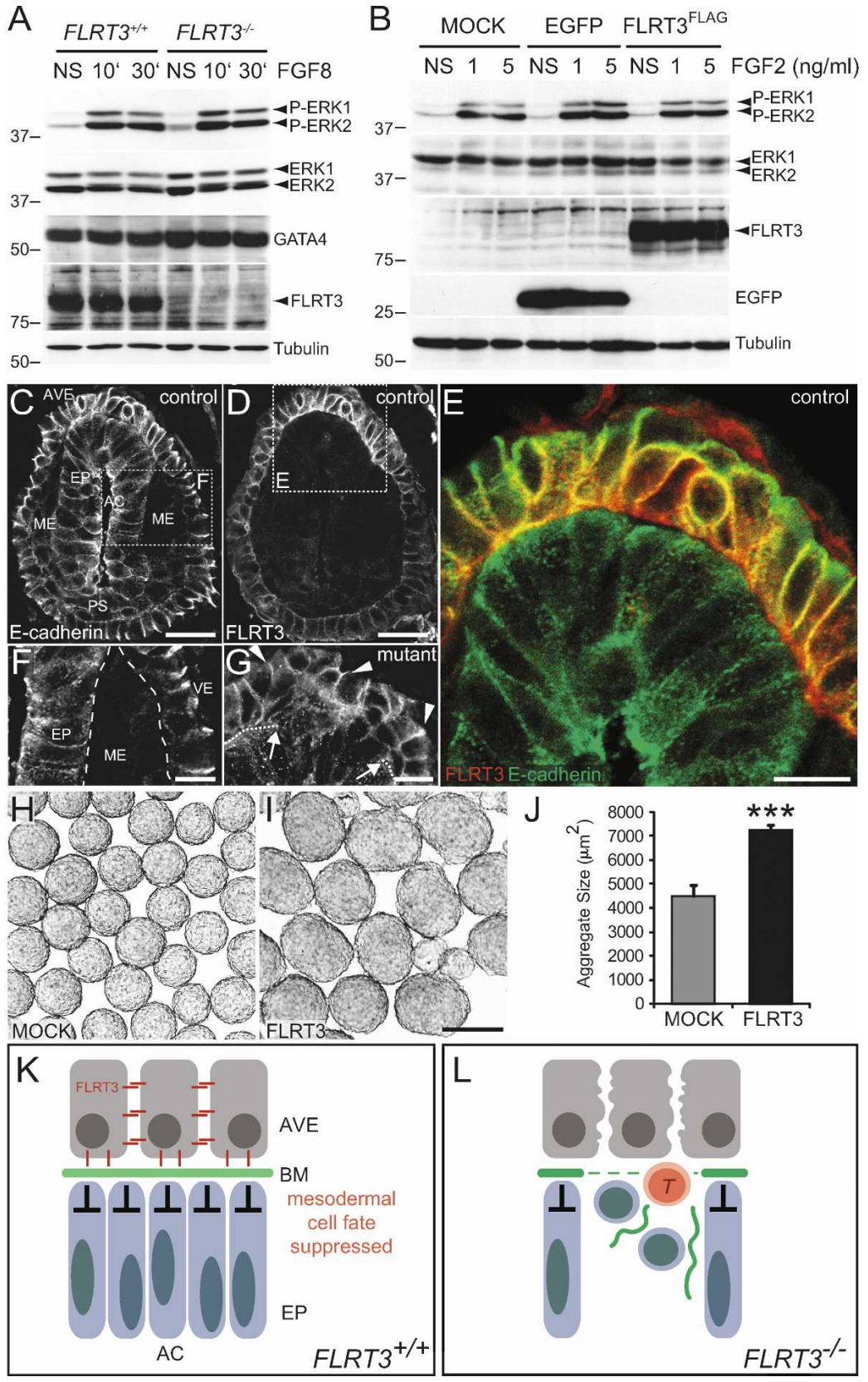


mal cells do not require FLRT3 to mediate FGF signaling. Moreover, overexpression of FLRT3 in HeLa cells also failed to increase ERK/MAPK phosphorylation upon FGF2 stimulation (Fig. 8B). Previous work had shown that Xenopus FLRT3 caused detachment of migrating equatorial cells via internalization of C-cadherin (Ogata et al. 2007). We therefore asked if FLRT3-positive AVE cells down-regulate E-cadherin expression like in migrating mesodermal cells at the mammalian PS (Burdsal et al. 1993). Double immunostaining for FLRT3 and E-cadherin of control MS stage embryos revealed high levels of E-cadherin in the lateral plasma membranes of FLRT3positive AVE cells (Fig. 8C-E). E-cadherin levels in AVE were much higher than in mesodermal cells (Fig. 8C,F), suggesting that migration of AVE cells did not require down-regulation of E-cadherin. Furthermore, levels and the cellular distribution of E-cadherin were apparently normal in $\mathrm{FLRT3}^{-/-}$embryos (Fig. 8G). Overexpression of FLRT3 caused homotypic cell sorting, suggesting that FLRT3 may act as a homophilic cell adhesion molecule (Karaulanov et al. 2006). We confirmed these observations (Supplemental Fig. S7), and we observed that cell aggregates of FLRT3-transfected cells were significantly larger than control aggregates (Fig. 8H-J). Altogether these results suggest that FLRT3 neither modulates FGFR signaling nor E-cadherin function but could mediate cell-cell adhesion in the AVE.

\section{Discussion}

The mouse AVE is an extraembryonic signaling center responsible for the proper orientation of the AP axis of the embryo: It provides positional cues by secreting inhibitors of posteriorizing activities and activators for epithelial cell intercalation movements (Rossant and Tam 2004; Voiculescu et al. 2007). In the present study, we describe a novel function of AVE cells in the anterior embryo. By deleting the gene encoding the transmembrane protein FLRT3, we observed that AVE cells were unable to support the formation and/or integrity of the BM both in vivo and in vitro. Subsequently, the subjacent epiblast cells lose their polarized state, migrate through the VE, and acquire features of EMT and mesodermal cell fate (see model in Fig. 8K,L). Hence, the AVE acts as a morphogenetic boundary to prevent EMT and mesoderm induction in the anterior epiblast through a mechanism that requires the stabilization of the BM. Although there have been observations that the AVE (or the chick hypoblast) may influence the behavior of nearby cells in the anterior embryo (Thomas and Beddington 1996; Foley et al. 2000), genetic evidence has been missing. Considering that AVE cells are highly migratory, such a morphogenetic role is rather unexpected.

In a recent study involving an independently generated FLRT3 knockout mouse line (Maretto et al. 2008), it was proposed that FLRT3 controls development of DE and directly participates in neural tube and ventral body wall closure. However, while we observed similar phenotypes in our $\mathrm{FLRT3}^{-/-}$embryos, the tetraploid rescue experiments and the observation of AVE defects before the DE reaches the anterior part of the embryo clearly indicates that FLRT3 functions mainly in the extraembryonic AVE. Our data suggest that the AVE plays an active role in keeping the integrity of the BM. The localization of BM breakdown in $\mathrm{FLRT3}^{-/-}$embryos correlates with the area of highest FLRT3 expression in the AVE. Importantly, signs of BM breakdown were often detected before the disruption of the AVE and never vice versa, indicating that effects on BM cannot simply be explained by the lack of AVE cells. In line with these observations, FLRT3 $^{-/-}$EBs displayed a disorganized BM with local disruptions that lead to the appearance of protrusions and an overall abnormal morphology. An alternative, but perhaps more complicated scenario would be that the $\mathrm{BM}$ is affected more globally, but provides enough stability for migrating mesoderm. In the anterior part of the embryo, the BM is not stable enough to withstand the morphogenetic movements, and epiblast cells are exposed.

In places of BM breakdown in $F L R T 3^{-/-}$embryos, epiblast cells displayed an EMT-like process as judged by loss of cell polarity, ingression, and up-regulation of EMT and mesodermal genes such as Eomes, T, and Fgf8. Eomes has been proposed to be a key regulator of EMT. In Eomes-deficient embryos, EMT and mesoderm delamination are completely blocked as a consequence of a failure of E-cadherin down-regulation during gastrulation (Arnold et al. 2008). However, it is not clear how Eomes regulates EMT and how it cooperates with Snail factors, central regulators of this process (Nieto 2002). We did not detect ectopic Snai1 expression in FLRT3-1embryos, suggesting that they do not acquire all features of EMT. Ectopic $T$ expression has been observed in $\mathrm{Cer1}^{-/-}$; Lefty $1^{-/-}$double-knockout embryos, indicating that reduced levels of Nodal inhibitors are sufficient to induce mesodermal fate in the anterior embryo (PereaGomez et al. 2002). However, the occurrence of ectopic $T$ expression was significantly lower in $C e r 1^{-/-}$; Lefty $1^{-/-}$ embryos (one out of four) than in FLRT3 ${ }^{-/-}$embryos (six out of 10). Moreover, we detected normal levels and patterns of Cer1, Lefty1, and Dkk1 in the AVE, and the expression domains of AP patterning markers in the epiblast, Wnt3 and Nodal, were not affected in FLRT3 $3^{-1}$ embryos. Taken together, these data suggest that mechanisms additional to the signaling functions of the AVE in AP patterning are operating to restrict EMT and mesoderm formation to the posterior epiblast. Support for this idea can be found in embryos deficient in Nap1, a regulator of WAVE-mediated actin dynamics. These embryos display AVE migration defects, and, as a consequence, inhibitors of posteriorizing signals are not delivered to the right place, leading to axis duplication (Rakeman and Anderson 2006). Importantly, Nap1 $1^{-/-}$embryos do not show the kind of ectopic $T$ expression or BM breakdown that we detect in $F L R T 3^{-/-}$embryos.

The percentage of $F L R T 3^{-/-}$embryos displaying ectopic $T$ expression and the percentage of embryos displaying BM defects are strongly correlated. There is increasing evidence that BMs are not simply structural scaffolds but also active regulators of cell migration, differentiation, 
or cell fate (Li et al. 2003). Expression of LN by the VE is required to stabilize the VE epithelium, to induce differentiation and polarization of the epiblast, and to prevent epiblast cells from transiting to a mesodermal fate (Murray and Edgar 2000, 2001; Fujiwara et al. 2007). Moreover, during avian gastrulation, BM breakdown appears to be the first recognizable step of EMT preceding the loss of tight junctions and apical polarity markers (Nakaya et al. 2008). Recently, a direct link between BM and the expression of EMT and mesodermal marker genes has been established (Fujiwara et al. 2007). Expression of Snai2 and Twist was up-regulated in $L A M C^{-/-}(\mathrm{LN} \gamma 1$ knockout) EBs that lack BM and fail to form a columnar epiblast. Interestingly, addition of exogenous LN rescued BM formation, epiblast differentiation and, most importantly, suppressed mesodermal markers. It is therefore plausible that BM regulates mesodermal cell fate in vivo and that disruption of the BM, like in FLRT3-/- embryos, leads to ectopic expression of mesodermal markers. The mechanisms of how exactly the BM controls mesodermal cell fate is unknown, but it could, for instance, act as a storage compartment regulating the availability and/or the presentation of mesodermal cell fate inhibitors as it was reported previously for growth factors (Aszodi et al. 2006).

How does FLRT3 regulate BM integrity in the AVE? In Xenopus embryos, XFLRT3 was shown to form a complex with FGF receptors and to enhance ERK/MAPK signaling (Bottcher et al. 2004). FGFR signaling, through the PI3K/Akt pathway, was shown to be important for LN synthesis and EB differentiation (Chen et al. 2000; Li et al. 2001). While we agree that mouse FLRT3 can interact with FGFR2 in transfected cells, our experiments suggest that this interaction is not relevant for the BM integrity of the AVE. Primary FLRT3 ${ }^{-/-}$endodermal cells displayed normal phosphorylation levels of ERK/MAPK and Akt upon FGF stimulation. In addition, $F L R T 3^{-/-}$ EBs showed normal LN expression and secretion (data not shown). These results are supported by the observation that $F L R T 3^{-/-}$embryos did not have defects in FGFR target gene expression after gastrulation (Maretto et al. 2008). Together, these results strongly indicate that FLRT3 does not regulate FGFR signaling in the AVE. However, they do not exclude a function for FLRT3 or other FLRT family members in modulating FGF signaling in other tissues at later stages of development.

The VE synthesizes and deposits BM components that are necessary for VE epithelia organization and epiblast polarization and differentiation (Harrisson et al. 1985; Murray and Edgar 2000, 2001). Although we did not observe a defect in synthesis or secretion of $\mathrm{LN}$ in $\mathrm{FLRT3}^{-/-}$ EBs, it is still possible that FLRT3 regulates the deposition of other BM components. Reduced transfer of these components in $\mathrm{FLRT3}^{-/-}$embryos could weaken the BM and cause it to rupture when tissue pressure rises. A precedent for such a situation was reported for perlecan, whose absence caused neuronal ectopias due to disruption of the BM surrounding the telencephalon /Costell et al. 1999). Alternatively, FLRT3 could be directly involved in BM polymerization and anchorage by binding to BM components or may confer competence to endodermal cells to anchor the BM. FLRT3 may also influence the expression and/or the function of proteases or protease inhibitors that affect BM stability and/or turnover. Recently, XFLRT3 was implicated in promoting deadhesion of involuting equatorial cells during gastrulation by promoting internalization of C-cadherin (Ogata et al. 2007). Our analysis of E-cadherin expression revealed, however, that normal AVE cells showed high levels of E-cadherin in the membrane (despite the presence of FLRT3) and that FLRT3-/- AVE cells did not show an increase in E-cadherin expression. Nevertheless, we observed that FLRT3 is able to promote homotypic cell sorting, suggesting that FLRT3 might directly or indirectly control cell-cell adhesion (see also Karaulanov et al. 2006). Therefore, one interesting possibility is that FLRT3 regulates BM integrity indirectly through the regulation of cell-cell adhesion, which is intimately interconnected with cell-matrix adhesion (Schock and Perrimon 2002). The exact mechanisms by which FLRT3 participates in the formation and/or stabilization of the BM would require further investigation. Nevertheless, whichever molecular mechanism it regulates, FLRT3 is the first transmembrane protein required for the morphogenetic role of the AVE and provides an entry point to study this important function in more detail.

\section{Materials and methods}

Antibody staining and confocal imaging

Cryosections were immunostained with the indicated antibodies: anti-FLRT3 (AF2795, R\&D Systems); anti-EGFP (Invitrogen $\mathrm{GmbH}$ ); anti-Sox2 (AB5603; Chemicon); anti-E-cadherin H-108 (sc-7870, Santa Cruz Biotechnologies); anti-LN (Sigma, L-9393); anti-perlecan (MAB1948 clone A7L6, Chemicon); anti-collagen IV (AB756P, Chemicon); anti-GATA-4 H-112 (sc-9053, Santa Cruz Biotechnologies); anti- $\beta$ lintegrin (MAB1997; Chemicon); anti-brachyury N-19 (sc-17743, Santa Cruz Biothechnologies); and anti-LN $\alpha 1$ (a gift from Rupert Timpl). Fluorescently labeled secondary antibodies with minimal cross-reaction were used (Jackson ImmunoResearch Laboratories). Stainings were analyzed by confocal microscopy (SP2, Leica Microsystems).

\section{$E B$ differentiation, endodermal cell culture, and biochemistry}

EBs were differentiated and processed as described previously (Montanez et al. 2007). Shape factor ( $\mathrm{SF}=4 \pi$ area/perimeter ${ }^{2}$ ) analysis was done using the MetaMorph software. To isolate endoderm cells, 5- to 6-d-old EBs were plated on fibronectincoated dishes. After $24 \mathrm{~h}$, the cells were gently trypsinized, collected, and allowed to settle down by gravity for $10 \mathrm{~min}$ at room temperature. The supernatant containing mainly endoderm cells was transferred into a fresh tube. The process was repeated two times to ensure complete removal of EBs. The resulting suspension was transferred to fibronectin-coated dishes. The next day, cells were serum starved for $\sim 10 \mathrm{~h}$ and stimulated with FGF. Total cell lysates were obtained as described previously (Egea et al. 2005). Western blot analysis: anti-P-p42/p44 ERK/MAPK (9101, Cell Signaling Technology); anti-p42/p44 ERK/MAPK (9102, Cell Signaling Technology); anti-tubulin (T9026, Sigma); anti-EGFP JL-8 (632381, Clontech); anti-AFP C-19 (sc-8108, Santa Cruz Biotechnologies); and Flag (ab-21536, Ab- 
cam). FLRT3 was detected using an antiserum (1134) obtained from rabbits immunized with a recombinant protein containing the intracellular domain of the mouse FLRT3 fused to the Glutathione-S-transferase.

\section{Cell sorting assay}

HEK293T cells were transiently transfected using calcium phosphate with pEGFP-N1 (Clontech) and pcDNA3 (MOCK) or pcDNA3-FLRT3 with a ratio of 1:2. Aggregation assays were performed similar to Karaulanov et al. (2006): Twenty-four hours after transfection, cells were dissociated in TE and $2.5 \times 10^{5}$ cells per milliliter aggregated in suspension with orbital shaking $(125 \mathrm{rpm})$ in BSA-coated $24-$ well plates at $37^{\circ} \mathrm{C}$ and $5 \% \mathrm{CO}_{2}$ for $48 \mathrm{~h}$. Photographs were taken with an inverted fluorescence microscope (Leica) connected to a cooled digital color camera (Olympus).

Additional Materials and Methods are in the Supplemental Material.

\section{Acknowledgments}

We thank the Klein group for fruitful discussion and support; $\mathrm{T}$. Rodriguez for Hex-GFP mice; T. Mäkinen, R. Dono, J. Partanen, A. Nieto, and M. Ros for in situ probes; E. Kramer, J. Schultz, A. Porthin, A. Mishra, and F. Helmbacher for help at earlier stages of this project; J. Bailey, A. Schaupp, and animal house staff for expert technical support; T. Mäkinen for critically reading the manuscript; and A. Nieto for discussion. S.Y. is a European Molecular Biology Organization and Japan Society for the Promotion of Science fellow. H.L. was supported by an EmmyNoether fellowship from the Deutsche Forschungsgemeinschaft. This work was in part funded by the Max-Planck Society. J.E. dedicates this work to Núria.

\section{References}

Albazerchi, A. and Stern, C.D. 2007. A role for the hypoblast (AVE) in the initiation of neural induction, independent of its ability to position the primitive streak. Dev. Biol. 301: 489-503.

Arnold, S.J., Hofmann, U.K., Bikoff, E.K., and Robertson, E.J. 2008. Pivotal roles for eomesodermin during axis formation, epithelium-to-mesenchyme transition and endoderm specification in the mouse. Development 135: 501-511.

Aszodi, A., Legate, K.R., Nakchbandi, I., and Fassler, R. 2006. What mouse mutants teach us about extracellular matrix function. Annu. Rev. Cell Dev. Biol. 22: 591-621.

Beddington, R.S. and Robertson, E.J. 1999. Axis development and early asymmetry in mammals. Cell 96: 195-209.

Bertocchini, F. and Stern, C.D. 2002. The hypoblast of the chick embryo positions the primitive streak by antagonizing nodal signaling. Dev. Cell 3: 735-744.

Bottcher, R.T., Pollet, N., Delius, H., and Niehrs, C. 2004. The transmembrane protein XFLRT3 forms a complex with FGF receptors and promotes FGF signalling. Nat. Cell Biol. 6: 38-44.

Burdsal, C.A., Damsky, C.H., and Pedersen, R.A. 1993. The role of E-cadherin and integrins in mesoderm differentiation and migration at the mammalian primitive streak. Development 118: 829-844.

Chen, Y., Li, X., Eswarakumar, V.P., Seger, R., and Lonai, P. 2000. Fibroblast growth factor (FGF) signaling through PI 3-kinase and Akt/PKB is required for embryoid body differentiation. Oncogene 19: 3750-3756.
Cho, E.A., Prindle, M.J., and Dressler, G.R. 2003. BRCT domaincontaining protein PTIP is essential for progression through mitosis. Mol. Cell. Biol. 23: 1666-1673.

Ciruna, B. and Rossant, J. 2001. FGF signaling regulates mesoderm cell fate specification and morphogenetic movement at the primitive streak. Dev. Cell 1: 37-49.

Costell, M., Gustafsson, E., Aszodi, A., Morgelin, M., Bloch, W., Hunziker, E., Addicks, K., Timpl, R., and Fassler, R. 1999. Perlecan maintains the integrity of cartilage and some basement membranes. J. Cell Biol. 147: 1109-1122.

Crossley, P.H. and Martin, G.R. 1995. The mouse Fgf8 gene encodes a family of polypeptides and is expressed in regions that direct outgrowth and patterning in the developing embryo. Development 121: 439-451.

Egea, J., Nissen, U.V., Dufour, A., Sahin, M., Greer, P., Kullander, K., Mrsic-Flogel, T.D., Greenberg, M.E., Kiehn, O., Vanderhaeghen, P., et al. 2005. Regulation of EphA 4 kinase activity is required for a subset of axon guidance decisions suggesting a key role for receptor clustering in Eph function. Neuron 47: 515-528.

Foley, A.C., Skromne, I., and Stern, C.D. 2000. Reconciling different models of forebrain induction and patterning: A dual role for the hypoblast. Development 127: 3839-3854.

Fujiwara, H., Hayashi, Y., Sanzen, N., Kobayashi, R., Weber, C.N., Emoto, T., Futaki, S., Niwa, H., Murray, P., Edgar, D., et al. 2007. Regulation of mesodermal differentiation of mouse embryonic stem cells by basement membranes. I. Biol. Chem. 282: 29701-29711.

Haines, B.P., Wheldon, L.M., Summerbell, D., Heath, J.K., and Rigby, P.W. 2006. Regulated expression of FLRT genes implies a functional role in the regulation of FGF signalling during mouse development. Dev. Biol. 297: 14-25.

Harrisson, F., Van Hoof, J., Vanroelen, C., and Vakaet, L. 1985. Transfer of extracellular matrix components between germ layers in chimaeric chicken-quail blastoderms. Cell Tissue Res. 239: 643-649.

Ishikawa, T., Yagyu, K., and Seguchi, H. 1986. A scanning electron microscopic study of the surface morphology of visceral endoderm and ectoderm in postimplantation mouse embryos. J. Electron Microsc. (Tokyo) 35: 185-194.

Karaulanov, E.E., Bottcher, R.T., and Niehrs, C. 2006. A role for fibronectin-leucine-rich transmembrane cell-surface proteins in homotypic cell adhesion. EMBO Rep. 7: 283-290.

Kimura-Yoshida, C., Nakano, H., Okamura, D., Nakao, K., Yonemura, S., Belo, J.A., Aizawa, S., Matsui, Y., and Matsuo, I. 2005. Canonical Wnt signaling and its antagonist regulate anterior-posterior axis polarization by guiding cell migration in mouse visceral endoderm. Dev. Cell 9: 639-650.

Lacy, S.E., Bonnemann, C.G., Buzney, E.A., and Kunkel, L.M. 1999. Identification of FLRT1, FLRT2, and FLRT3: A novel family of transmembrane leucine-rich repeat proteins. Genomics 62: 417-426.

Li, X., Chen, Y., Scheele, S., Arman, E., Haffner-Krausz, R., Ekblom, P., and Lonai, P. 2001. Fibroblast growth factor signaling and basement membrane assembly are connected during epithelial morphogenesis of the embryoid body. J. Cell Biol. 153: 811-822.

Li, S., Edgar, D., Fassler, R., Wadsworth, W., and Yurchenco, P.D. 2003. The role of laminin in embryonic cell polarization and tissue organization. Dev. Cell 4: 613-624.

Lu, C.C., Brennan, J., and Robertson, E.J. 2001. From fertilization to gastrulation: Axis formation in the mouse embryo. Curr. Opin. Genet. Dev. 11: 384-392.

Maretto, S., Muller, P.S., Aricescu, A.R., Cho, K.W., Bikoff, E.K., and Robertson, E.J. 2008. Ventral closure, headfold fusion and definitive endoderm migration defects in mouse em- 
bryos lacking the fibronectin leucine-rich transmembrane protein FLRT3. Dev. Biol. 318: 184-193.

Montanez, E., Piwko-Czuchra, A., Bauer, M., Li, S., Yurchenco, P., and Fassler, R. 2007. Analysis of integrin functions in peri-implantation embryos, hematopoietic system, and skin. Methods Enzymol. 426: 239-289.

Murray, P. and Edgar, D. 2000. Regulation of programmed cell death by basement membranes in embryonic development. J. Cell Biol. 150: 1215-1221.

Murray, P. and Edgar, D. 2001. Regulation of the differentiation and behaviour of extra-embryonic endodermal cells by basement membranes. J. Cell Sci. 114: 931-939.

Nakaya, Y., Sukowati, E.W., Wu, Y., and Sheng, G. 2008. RhoA and microtubule dynamics control cell-basement membrane interaction in EMT during gastrulation. Nat. Cell Biol. 10: 765-775.

Nieto, M.A. 2002. The snail superfamily of zinc-finger transcription factors. Nat. Rev. 3: 155-166.

Ogata, S., Morokuma, J., Hayata, T., Kolle, G., Niehrs, C., Ueno, N., and Cho, K.W. 2007. TGF- $\beta$ signaling-mediated morphogenesis: Modulation of cell adhesion via cadherin endocytosis. Genes \& Dev. 21: 1817-1831.

Perea-Gomez, A., Vella, F.D., Shawlot, W., Oulad-Abdelghani, M., Chazaud, C., Meno, C., Pfister, V., Chen, L., Robertson, E., Hamada, H., et al. 2002. Nodal antagonists in the anterior visceral endoderm prevent the formation of multiple primitive streaks. Dev. Cell 3: 745-756.

Rakeman, A.S. and Anderson, K.V. 2006. Axis specification and morphogenesis in the mouse embryo require Nap1, a regulator of WAVE-mediated actin branching. Development 133: 3075-3083.

Rodriguez, T.A., Casey, E.S., Harland, R.M., Smith, J.C., and Beddington, R.S. 2001. Distinct enhancer elements control Hex expression during gastrulation and early organogenesis. Dev. Biol. 234: 304-316.

Rossant, J. and Tam, P.P. 2004. Emerging asymmetry and embryonic patterning in early mouse development. Dev. Cell 7: $155-164$.

Schock, F. and Perrimon, N. 2002. Molecular mechanisms of epithelial morphogenesis. Annu. Rev. Cell Dev. Biol. 18: 463-493.

Simeone, A., Acampora, D., Mallamaci, A., Stornaiuolo, A., D'Apice, M.R., Nigro, V., and Boncinelli, E. 1993. A vertebrate gene related to orthodenticle contains a homeodomain of the bicoid class and demarcates anterior neuroectoderm in the gastrulating mouse embryo. EMBO J. 12: 2735-2747.

Srinivas, S. 2006. The anterior visceral endoderm-turning heads. Genesis 44: 565-572.

Srinivas, S., Rodriguez, T., Clements, M., Smith, J.C., and Beddington, R.S. 2004. Active cell migration drives the unilateral movements of the anterior visceral endoderm. Development 131: 1157-1164.

Tam, P.P. and Loebel, D.A. 2007. Gene function in mouse embryogenesis: Get set for gastrulation. Nat. Rev. Genet. 8: 368-381.

Tam, P.P. and Meier, S. 1982. The establishment of a somitomeric pattern in the mesoderm of the gastrulating mouse embryo. Am. J. Anat. 164: 209-225.

Tam, P.P. and Rossant, J. 2003. Mouse embryonic chimeras: Tools for studying mammalian development. Development 130: 6155-6163.

Thiery, J.P. and Sleeman, J.P. 2006. Complex networks orchestrate epithelial-mesenchymal transitions. Nat. Rev. 7: 131142 .

Thomas, P. and Beddington, R. 1996. Anterior primitive endoderm may be responsible for patterning the anterior neural plate in the mouse embryo. Curr. Biol. 6: 1487-1496.

Viebahn, C., Mayer, B., and Miething, A. 1995. Morphology of incipient mesoderm formation in the rabbit embryo: A lightand retrospective electron-microscopic study. Acta Anat. (Basel) 154: 99-110.

Voiculescu, O., Bertocchini, F., Wolpert, L., Keller, R.E., and Stern, C.D. 2007. The amniote primitive streak is defined by epithelial cell intercalation before gastrulation. Nature 449: 1049-1052.

Wood, H.B. and Episkopou, V. 1999. Comparative expression of the mouse Sox1, Sox2 and Sox3 genes from pre-gastrulation to early somite stages. Mech. Dev. 86: 197-201.

Yamamoto, M., Saijoh, Y., Perea-Gomez, A., Shawlot, W., Behringer, R.R., Ang, S.L., Hamada, H., and Meno, C. 2004. Nodal antagonists regulate formation of the anteroposterior axis of the mouse embryo. Nature 428: 387-392. 


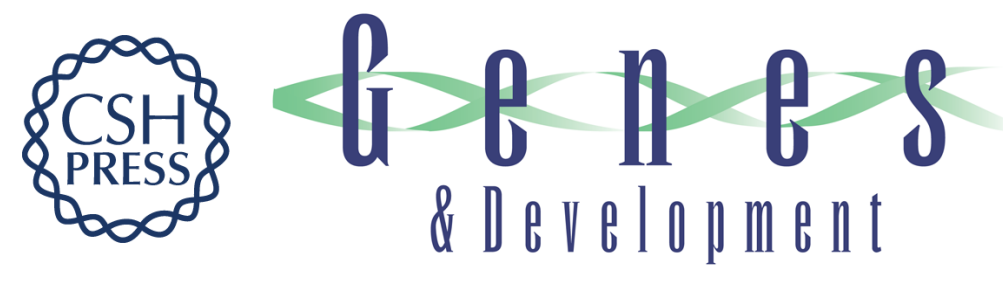

\section{Genetic ablation of FLRT3 reveals a novel morphogenetic function for the anterior visceral endoderm in suppressing mesoderm differentiation}

Joaquim Egea, Christian Erlacher, Eloi Montanez, et al.

Genes Dev. 2008, 22:

Access the most recent version at doi:10.1101/gad.486708

Supplemental Material

References

License

Email Alerting

Service
http://genesdev.cshlp.org/content/suppl/2008/11/18/22.23.3349.DC1

This article cites 48 articles, 14 of which can be accessed free at: http://genesdev.cshlp.org/content/22/23/3349.full.html\#ref-list-1

Receive free email alerts when new articles cite this article - sign up in the box at the top right corner of the article or click here.

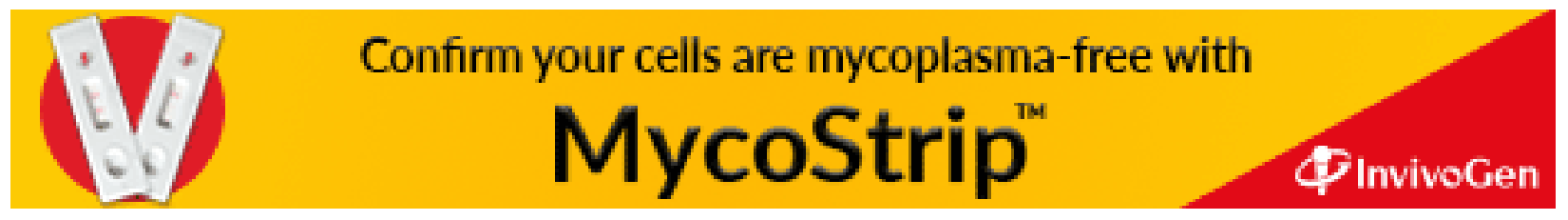

